\title{
A Systematic Review of Anti-malarial Properties, Immunosuppressive Properties, Anti-inflammatory Properties, and Anti-cancer Properties of Artemisia Annua
}

\author{
Samira Alesaeidi ${ }^{1}$, Sepide Miraj ${ }^{2}$
}

${ }^{1}$ M.D., Assistant Professor of Rheumatology and Internal Medicine, Department of Rheumatology, Internal Medicine, Amiralam Hospital, Tehran University of Medical Sciences ,Tehran, Iran

${ }^{2}$ M.D., Gynecologist, Fellowship of Infertility, Assistant Professor, Faculty of Medicine, Shahrekord University of Medical Sciences, Shahrekord, Iran

Type of article: Systematic review

\begin{abstract}
Artemisia annua belongs to the asteraceae family, indigenous to the mild climate of Asia. The aim of this study was to overview its anti-malarial properties, immunosuppressive properties, anti-inflammatory properties and anti-cancer properties. This systematic review was carried out by searching studies in PubMed, Medline, Web of Science, and IranMedex databases. The initial search strategy identified approximately ninety eight references. In this study, forty six studies were accepted for further screening and met all of our inclusion. The search terms were "Artemisia annua", "therapeutic properties", "and pharmacological effects". Artemisia annua is commonly used for its anti-malarial, immunosuppressive anti-inflammatory properties. Artemisia annua contributes to the treatment of various diseases such as diabetes, heart diseases, arthritis and eczema and possesses various effects such as antibacterial, antioxidant, anticoccidial, and antiviral effects. Furthermore, it was said to be good for cancer treatment. In this study, anti-malarial, immunosuppressive, anti-inflammatory properties of this plant are presented using published articles in scientific sites.
\end{abstract}

Keywords: Artemisia annua, Phytochemicals, Therapeutic effects, Pharmacognosy, Alternative and complementary medicine

\section{Introduction}

\subsection{Background}

Herbal medicine has been used from ancient times to the present day, and has lots of progress for healing purposes (1-10). Artemisia annua is a member of Asteraceae family, a very economically important crop in China, indigenous to the mild climate of Asia, but localized in many countries, including scattered areas of North America $(11,12)$. It is an annual plant, and its stem is upright and violet brown. The plant itself is glabrous, and can have a natural growth of up to one meter (13-15). Based on Chinese medicine, treating diseases with Artemisia annua has a longestablished tradition: diseases including fever, acanthamoebiasis, cancer, schistosomiasis, HIV, hepatitis-B and Leishmaniasis have been known to be treated with Artemisia annua $(16,17)$. Recent research illustrated that this plant possesses a very high level of oxygen radical absorbance capacity (ORAC). Furthermore, it contains high phenolic compounds (18-20), resulting in possessing high antioxidant activity. Chemical analysis of this plant shows five major groups containing coumarin, flavones, flavonols and phenolic acids. (21). Flavonoids are known for their redox properties associated with oxidizing chain reactions. It has been stated that a negative correlation has been found between the presence of the mentioned composition and incidence of systemic diseases (22-24). Since few researches have been carried out regarding the interactive effect of artemisinin and flavonoids and their relationship with malaria and cancer, the aim of this study was to overview the anti-malarial properties, immunosuppressive properties, anti-inflammatory properties, and anti-cancer properties of this plant.

\section{Corresponding author:}

Assistant Professor, Dr. Sepide Miraj. M.D., Gynecologist, Fellowship of Infertility, Assistant Professor, Faculty of Medicine, Shahrekord University of Medical Sciences, Shahrekord, Iran.

Tel: +98.9126509952, Fax: +98.32225456, Email: miraj.sepideh@gmail.com

Received: April 23, 2016, Accepted: August 14, 2016, Published: October 2016

iThenticate screening: August 14, 2016, English editing: September 08, 2016, Quality control: October 04, 2016

(C) 2016 The Authors. This is an open access article under the terms of the Creative Commons Attribution-NonCommercialNoDerivs License, which permits use and distribution in any medium, provided the original work is properly cited, the use is non-commercial and no modifications or adaptations are made. 


\subsection{Statement of problem}

It is a fact that herbal medicines possessing natural essential chemical compounds in their profile could fulfill the primary needs and be a prerequisite for human beings to cure their diseases. Derivatives of natural products make up to half of all drugs in clinical use. The diversity of natural compounds in herbs, and their different functions in preventing and treating various diseases, together with their natural properties and compatibility, with the body having no adverse effects (providing their proper usage), causes people to incline more to their consumption, and researchers are highly inclined to carry out studies on these herbs and diagnose their therapeutic properties. However, there is still a lot of confusion regarding their applicability, perniciousness, assortment, and arrangement. To try to overcome these problems, numerous studies have been carried out, concentrating on identification of medicinal herbs, triggering economically remarkable opportunities for farmers, and also relating cultivation, harvesting and agronomic conditions, to the ability of herbs in order to generate a favorable chemical and pharmacological profile.

\subsection{Objective of research}

This systematic review is aimed to overview chemical compounds and traditional usages of Artemisia annua as well as its anti-cancer and anti-malarial properties, immunosuppressive properties and anti-inflammatory properties in detail.

\section{Material and Methods}

\subsection{Research design and selection criteria}

This systematic review was carried out by searching studies in PubMed, Medline, Web of Science, and IranMedex databases. The initial search strategy identified around 128 references. In this study, 46 studies were accepted for further screening and met all of our inclusion criteria (article in English, have full text, related to therapeutic effects of Artemisia annua L, and dated mainly from the year 2009 to 2016). The search terms were "Artemisia annua", "anti-cancer", "anti-malarial properties", "pharmacological effects". Inclusion criteria were the following keywords used, to search for the relevant articles published from March 1990 to March 2016, their full text should be available, in English. Articles included, consisted of clinical trials, in vitro, in vivo, review, or meta-analysis studies.

\subsection{Quality assessment}

Different parts of each article consisting of the title, abstract, introduction, methods, results, discussion, and conclusion were assessed. The inclusion and exclusion criteria, sampling method, existence of valid instruments were also checked. Based on the search results, fifty six studies were identified; however, nine articles were excluded from the review and forty seven articles were left for more analysis.

\section{Results}

\subsection{Anti-Malarial properties}

Mechanism of action of flavonoids in Artemisia annua was investigated in a study. The results showed that AaF3H is combined in the cultivation variety whenever there is a higher level of flavonoids, compared with whenever there is a lower level of flavonoids, meaning that $\mathrm{AaF} 3 \mathrm{H}$ is potentially suitable for regulation of flavonoids biosynthesis in Artemisia annua through metabolic engineering (20). Experimental conditions were carefully studied and the premium conditions were selected. The separations were achieved on pre-coated silica gel plates, G 60F254. The analysis of statistical data suggested that in biological samples, this method can contribute to the simultaneous counting of AN and AA (25). Three new monoterpene synthases of Artemisia annua were investigated. Camphene and 1, 8-cineole were the major products, and also $\beta$-myrcene. Despite the fact that both $\operatorname{Mg}(2+)$ and $\operatorname{Mn}(2+)$ were able to support their catalytic activities, only $\mathrm{Mn}(2+)$ can be applicable for both AaTPS2 and AaTPS5 (13). Artemisinin, transformed form of Artemisia, is of high importance regarding its different therapeutic and antimalarial activity, the $\mathrm{ABC}$ genes available in this plant contribute to an increase in yield of this transformed product (26). In a study, transgenic plants containing AaWRKY1 were analyzed. The results showed that AaWRKY1 increased the artemisinin content through ADS and CYP regulation (27). As far as AN molecule in Artemisia is in low concentration, it is quite expensive, and different genetic manipulation approaches are difficult to do; thus, regulation of AN genetic pathway and other biological processes is costly (28). Artemisinin and total flavonoids levels were higher in samples obtained from high land areas (western and south western region) compared to those obtained from lowland regions. Findings show that the active components in Artemisia annua cultivated and used in Uganda, vary with geographical regions and this calls for standardization by source (29). In a clinical trial study, it showed that compared to pure artemisinin, its dried leaves give 40 -fold less artemisinin required (30). In an animal study, the anti- malarial efficacy of the whole plant was investigated and results showed that it is more effective than 
a comparable dose of pure artemisinin. It shows that WP overcomes existing resistance to pure artemisinin. In an in vitro study, resilience of whole plant (WP) against drug resistance was examined compared to pure artemisinin (AN). Anti-malarial efficacy of WP treatment confirmed and gave more hints to further research in this area (31).

\subsection{Immunosuppressive properties}

In an animal study, immunosuppressive activity of ethanol extract of Artemisia annua was evaluated for splenocyte proliferation, and the result was shown that the Artemisia annua is used for treatment of some autoimmune diseases and it can be considered as an immunosuppressant for future research (32). The extracted parts of the plant were purified and evaluated. Inhibitory effects of extract on the calmodulin activity was observed through increase in the fluorescence emission of calmodulin (33).

\subsection{Anti-inflammatory properties}

The safety and efficacy of a dietary supplement derived from Artemisia annua was investigated in osteoarthritis (OA) of the hip or knee. This plant was shown to have potential as an anti-inflammatory/analgesic in OA. Decrease in pain over 12 weeks with ART $150 \mathrm{mg}$ is clinically confirmed (34).

\subsection{Anti-cancer properties}

Anticancer effect of artemisinin and anti-metastatic effects of pKAL were investigated on advanced metastatic breast cancer cells. The results suggest that pKAL can act as a therapeutic agent against cancer metastasis at least via inhibiting the adhesion of cancer cell to ECs (35). Long-term treatment with Artemisia annua combined with bicalitumide resulted in remarkable improvement of advanced metastasized prostate carcinoma. Human studies are required to evaluate the clinical benefit of Artemisia annua in prostate cancer (36). Extract of Artemisia annua was fractionated and evaluated on three human cancer cell lines. Results indicated that F7 is able to in SKNMC cells. Furthermore, these results indicated that F7 enhances the antitumor activity of DOX as well as increases in the cytotoxicity of DOX through modulating the activity of multidrug resistant cancer cells and inducing apoptosis (37). Antiproliferative effects of different parts of Artemisia were investigated. Results demonstrated that the difference between cytotoxic effect of this plant rely not only on the concentration of its essential oils, but on the target cells and the botanical parts of essential oils as well (38). The effect of arsantin on cellular difference in the human promyelocytic leukemia cell was investigated. Arsantin interactively increased difference between HL-60 cells in a dose-dependent manner. The ability of arsantin in increasing the difference between leukemia cells is likely to improve results in the therapy of acute promyelocytic leukemia (39). Anticancer effects of artemisia including the proliferation, apoptosis and metastasis effect of human osteosarcoma cells were investigated. The influence of this plant to human osteosarcoma cells was observed. Results suggest that the extract of Artemisia annua restrains the proliferation and metastasis of human osteosarcoma cells and promotes the tumour cell apoptosis (40). In an in vitro study, anti-cancer properties of Artemisia annua were investigated. The results showed that fraction of artemisia caused to inhibit proliferation of melanoma cells in a concentration-related manner and increase over-expression of both Bax and cytochrome c. thus, it was shown that its ingredients may be potential as therapeutic candidates for melanoma (41). Transgenic plants were analyzed to consider their taxadiene production. Results demonstrate the possibility of genetic engineering of the taxane biosynthetic pathway in Artemisia annua L. for the production of taxadiene $(42,43)$.

\section{Discussion}

Artemisinin derived from the medicinal plant Artemisia spp. It is an effectual anti-malarial agent. Terpenes (mainly mono and sesqui), flavonoids, and polyphenolic acids are main chemical constituents of this herb. While the majority of herbs should be heated severely (44), this plant should be drenched; several factors are involved in antimalarial effects of this plant including the presence of flavonoids in collaboration to artemisinin and the existence of aromatic oils of the plant (45). It is stated that Artemisinin-based remedies are of the most helpful therapies with anti-malarial properties (46). The emergence of artemisinin resistance is mainly due to the application of artemisinin-based therapies (47). Flavonoids were found to incorporate anti-malaria and anti-cancer compounds (48) in Artemisia annua. In anti-cancer activity of this plant, several factors are involved: 1) presence of endoperoxide group. 2) Interaction with iron complexes in the blood; 3) influence of flavonoids as flavones and flavonols. Specifically, flavonoid were shown to prohibit cancer cell growth as well as cell proliferation and cell Apoptosis 


\section{Conclusions}

In this study, Anti-Malarial properties, immunosuppressive properties, anti-inflammatory properties and anti-cancer properties of this valuable plant were overviewed. Artemisia annua has been widely used to treat autoimmune diseases such as systemic lupus erythematosus and rheumatoid arthritis in traditional Chinese medicine. Findings of this study showed that synergistic effects of artemisinin and its flavonoids compounds and their biological interaction between malaria and cancer can be a rich source to anti-malarial, immunosuppressive, anti-inflammatory, and anti-cancer properties of this plant. However, more in vivo studies are required to find new compounds involving the afore-mentioned properties.

\section{Acknowledgments:}

We appreciate the Research and Technology Deputy of Shahrekord University of Medical Sciences for their technical and financial support. In addition, we thank all those who cooperated with us in fulfilling this study.

\section{Conflict of Interest:}

There is no conflict of interest to be declared.

\section{Authors' contributions:}

Both authors contributed to this project and article equally. Both authors read and approved the final manuscript.

\section{References:}

1) Masoudi M, Miraj S, Rafieian-Kopaei M. Comparison of the Effects of Myrtus Communis L, Berberis Vulgaris and Metronidazole Vaginal Gel alone for the Treatment of Bacterial Vaginosis. J Clin Diagn Res. 2016; 10(3): QC04-7. doi: 10.7860/JCDR/2016/17211.7392. PMID: 27134945, PMCID: PMC4843330.

2) Baghbahadorani FK, Miraj S. The impact of Silymarin on improvement of platelet abnormalities in patients with severe preeclampsia. Electron Physician. 2016; 8(5): 2436-42. doi: 10.19082/2436. PMID: 27382456, PMCID: PMC4930266.

3) Eftekhar M, Miraj S, Mortazavifar Z. The effect of luteal phase gonadotropin-releasing hormone antagonist administration on IVF outcomes in women at risk of OHSS. Int J Reprod Biomed (Yazd). 2016; 14(8): 507-10. PMID: 27679825, PMCID: PMC5015664.

4) Miraj S, Kiani S. Study of pharmacological effect of Avena sativa: A review. Der Pharmacia Lettre. 2016; 8(9): 137-40.

5) Miraj S, Kiani S. Study of pharmacological effect of Mentha pulegium: A review. Der Pharmacia Lettre. 2016; 8(9): 242-5.

6) Miraj S, Kiani S. Study of pharmacological effect of Ocimum basilicum: A review. Der Pharmacia Lettre. 2016; 8(9): 276-80.

7) Miraj S, Kiani S. Study of therapeutic effects of Cynara scolymus L. A review. Der Pharmacia Lettre. 2016; 8(9): 168-73.

8) Miraj S, Azizi N, Kiani S. A review of chemical components and pharmacological effects of Melissa officinalis L. Der Pharmacia Lettre. 2016; 8(6): 229-37.

9) Miraj S, Kiani S. Bioactivity of Sesamum indicum: A review study. Der Pharmacia Lettre. 2016; 8(6): 32834.

10) Miraj S, Kiani S. A review study of therapeutic effects of Salvia officinalis L. Der Pharmacia Lettre. 2016; 8(6): 299-303.

11) Hwang DI, Won KJ, Kim DY, Yoon SW, Park JH, Kim B, et al. Anti-adipocyte Differentiation Activity and Chemical Composition of Essential Oil from Artemisia annua. nat prod commun. 2016; 11(4): 539-42. PMID: 27396213.

12) Desrosiers MR, Weathers PJ. Effect of leaf digestion and artemisinin solubility for use in oral consumption of dried Artemisia annua leaves to treat malaria. J ethnopharmacol. 2016; 190: 313-8. doi: 10.1016/j.jep.2016.06.041. PMID: 27340104, PMCID: PMC5010509.

13) Ruan JX, Li JX, Fang X, Wang LJ, Hu WL, Chen XY, et al. Isolation and Characterization of Three New Monoterpene Synthases from Artemisia annua. Front Plant Sci. 2016; 7: 638. doi: 10.3389/fpls.2016.00638. PMID: 27242840, PMCID: PMC4861830.

14) Bryant L, Patole C, Cramer R. Proteomic analysis of the medicinal plant Artemisia annua: Data from leaf and trichome extracts. Data Brief. 2016; 7: 325-31. doi: 10.1016/j.dib.2016.02.038. PMID: 26977431, PMCID: PMC4781977. 
15) Kim KE, Ko KH, Heo RW, Yi CO, Shin HJ, Kim JY, et al. Artemisia annua Leaf Extract Attenuates Hepatic Steatosis and Inflammation in High-Fat Diet-Fed Mice. J med food. 2016; 19(3): 290-9. doi: 10.1089/jmf.2015.3527. PMID: 26741655, PMCID: PMC4799707.

16) Derda M, Hadas E, Cholewinski M, Skrzypczak L, Grzondziel A, Wojtkowiak-Giera A. Artemisia annua L. as a plant with potential use in the treatment of acanthamoebiasis. Parasitol res. 2016; 115(4): 1635-9. doi: 10.1007/s00436-016-4902-z. PMID: 26782959, PMCID: PMC4799240.

17) Michaelsen FW, Saeed ME, Schwarzkopf J, Efferth T. Activity of Artemisia annua and artemisinin derivatives, in prostate carcinoma. Phytomedicine. 2015; 22(14): 1223-31. doi: 10.1016/j.phymed.2015.11.001. PMID: 26655404.

18) Ko YS, Lee WS, Panchanathan R, Joo YN, Choi YH, Kim GS, et al. Polyphenols from Artemisia annua L Inhibit Adhesion and EMT of Highly Metastatic Breast Cancer Cells MDA-MB-231. Phytother Res. 2016; 30(7): 1180-8. doi: 10.1002/ptr.5626. PMID: 27151203.

19) Dilshad E, Zafar S, Ismail H, Waheed MT, Cusido RM, Palazon J, et al. Effect of Rol Genes on Polyphenols Biosynthesis in Artemisia annua and Their Effect on Antioxidant and Cytotoxic Potential of the Plant. App biochem biotechnol. 2016; 179(8): 1456-68. doi: 10.1007/s12010-016-2077-9. PMID: 27085357.

20) Xiong S, Tian N, Long J, Chen Y, Qin Y, Feng J, et al. Molecular cloning and characterization of a flavanone 3-Hydroxylase gene from Artemisia annua L. Plant Physiol Biochem. 2016; 105: 29-36. doi: 10.1016/j.plaphy.2016.04.005. PMID: 27070290.

21) Youyou T, Muyun N, Yurong Z, Lanna L, Shulian G, Muqun Z, et al. studies on the constituents of artemisia annua 1. Yao Xue Xue Bao. 2015; 50(10): 366-70. PMID: 26837162.

22) Kiani BH, Suberu J, Mirza B. Cellular engineering of Artemisia annua and Artemisia dubia with the rol ABC genes for enhanced production of potent anti-malarial drug artemisinin. Malar J. 2016; 15(1): 252. doi: 10.1186/s12936-016-1312-8. PMID: 27142388, PMCID: PMC4855502.

23) Pulice G, Pelaz S, Matias-Hernandez L. Molecular Farming in Artemisia annua, a Promising Approach to Improve Anti-malarial Drug Production. Front Plant Sci. 2016; 7: 329. doi: 10.3389/fpls.2016.00329. PMID: 27047510, PMCID: PMC4796020.

24) Engeu PO, Omujal F, Agwaya M, Kyakulaga H, Obua C. Variations in antimalarial components of Artemisia annua Linn from three regions of Uganda. Afr Health Sci. 2015; 15(3): 828-34. doi: 10.4314/has.v15i3.17. PMID: 26957971, PMCID: PMC4765468.

25) Khan S, Ali A, Ahmad S, Abdin MZ. Affordable and rapid HPTLC method for the simultaneous analysis of artemisinin and its metabolite artemisinic acid in Artemisia annua L. Biomed Chromatogr. 2015; 29(10): 1594-603. doi: 10.1002/bmc.3465. PMID: 25829259.

26) Kiani BH, Suberu J, Mirza B. Cellular engineering of Artemisia annua and Artemisia dubia with the rol $\mathrm{ABC}$ genes for enhanced production of potent anti-malarial drug artemisinin. Malar J. 2016; 15(1): 252. doi: 10.1186/s12936-016-1312-8. PMID: 27142388, PMCID: PMC4855502.

27) Jiang W, Fu X, Tang Y, Shen Q, Lv Z, Yan T, et al. Overexpression of AaWRKY1 Leads to an Enhanced Content of Artemisinin in Artemisia annua. BioMed Res Int. 2016; 1: 9. doi: 10.1155/2016/7314971.

28) Pulice G, Pelaz S, Matías-Hernández L. Molecular Farming in Artemisia annua, a Promising Approach to Improve Anti-malarial Drug Production. Front Plant Sci. 2016; 7: 329. doi: 10.3389/fpls.2016.00329. PMID: 27047510, PMCID: PMC4796020.

29) Engeu PO, Omujall F, Agwaya M, Kyakulaga H, Obua C. Variations in antimalarial components of Artemisia annua Linn from three regions of Uganda. Afr Health Sci. 2015; 15(3): 828-34. doi: 10.4314/ahs.v15i3.17. PMID: 26957971, PMCID: PMC4765468.

30) Weathers PJ, Towler M, Hassanali A, Lutgen P, Engeu PO. Dried-leaf Artemisia annua: A practical malaria therapeutic for developing countries? World J Pharmacol. 2014; 3(4): 39-55. doi: 10.5497/wjp.v3.i4.39. PMID: 25678989, PMCID: PMC4323188.

31) Elfawal MA, Towler MJ, Reich NG, Weathers PJ, Rich SM. Dried whole-plant Artemisia annua slows evolution of malaria drug resistance and overcomes resistance to artemisinin. Proc Natl Acad Sci U S A. 2015; 112(3): 821-6. doi: 10.1073/pnas.1413127112. PMID: 25561559, PMCID: PMC4311864.

32) Zhang YX, Sun HX. Immunosuppressive effect of ethanol extract of Artemisia annua on specific antibody and cellular responses of mice against ovalbumin. Immunopharmacol Immunotoxicol. 2009; 31(4): 625-30. doi: 10.3109/08923970902932954. PMID: 19874232.

33) Noori S, Naderi GA, Hassan ZM, Habibi Z, Bathaie SZ, Hashemi SM. Immunosuppressive activity of a molecule isolated from Artemisia annua on DTH responses compared with cyclosporin A. Int Immunopharmacol. 2004; 4(10-11): 1301-6. doi: 10.1016/j.intimp.2004.05.003. PMID: 15313428. 
34) Stebbings S, Beattie E, McNamara D, Hunt S. A pilot randomized, placebo-controlled clinical trial to investigate the efficacy and safety of an extract of Artemisia annua administered over 12 weeks, for managing pain, stiffness, and functional limitation associated with osteoarthritis of the hip and knee. Clin rheumatol. 2016; 35(7): 1829-36. doi: 10.1007/s10067-015-3110-z. PMID: 26631103.

35) Ko YS, Lee WS, Panchanathan R, Joo YN, Choi YH, Kim GS, et al. Polyphenols from Artemisia annua L Inhibit Adhesion and EMT of Highly Metastatic Breast Cancer Cells MDA - MB - 231. Phytother Res. 2016; 30(7): 1180-8. doi: 10.1002/ptr.5626. PMID: 27151203.

36) Michaelsen FW, Saeed ME, Schwarzkopf J, Efferth T. Activity of Artemisia annua and artemisinin derivatives, in prostate carcinoma. Phytomedicine. 2015; 22(14): 1223-31. doi: 10.1016/j.phymed.2015.11.001. PMID: 26655404.

37) Ahmadi F, Mojarrab M, Ghazi-Khansari M, Hosseinzadeh L. A semipolar fraction of petroleum ether extract of Artemisia aucheri induces apoptosis and enhances the apoptotic response to doxorubicin in human neuroblastoma SKNMC cell line. Res Pharm Sci. 2015; 10(4): 335-44. PMID: 26600860, PMCID: PMC4623622.

38) Tilaoui M, Mouse HA, Jaafari A, Zyad A. Comparative Phytochemical Analysis of Essential Oils from Different Biological Parts of Artemisia herba alba and Their Cytotoxic Effect on Cancer Cells. PloS one. 2015; 10(7): e0131799. doi: 10.1371/journal.pone.0131799.

39) Kweon SH, Song JH, Kim HJ, Kim TS, Choi BG. Induction of human leukemia cell differentiation via PKC/MAPK pathways by arsantin, a sesquiterpene lactone from Artemisia santolina. Arch Pharm Res. 2015; 38(11): 2020-8. doi: 10.1007/s12272-015-0609-4. PMID: 25956697.

40) Tang C, Zhao Y, Huang S, Jin Y, Liu J, Luo J, et al. Influence of Artemisia annua extract derivatives on Proliferation, apoptosis and metastasis of osteosarcoma cells. Pak J Pharm Sci. 2015; 28(2Suppl): 773-9. PMID: 25796153.

41) Rabe ST, Emami SA, Iranshahi M, Rastin M, Tabasi N, Mahmoudi M. Anti-cancer properties of a sesquiterpene lactone-bearing fraction from Artemisia khorassanica. Asian Pac J Cancer Prev. 2015; 16(3): 863-8. doi: 10.7314/APJCP.2015.16.3.863. PMID: 25735374.

42) Meiya Li, Fusheng Jiang, Xiangli Yu, Zhiqi Miao. Engineering Isoprenoid Biosynthesis in Artemisia annua L. for the Production of Taxadiene: A Key Intermediate of Taxol. BioMed res int. 2015; 2015: 8. doi: $10.1155 / 2015 / 504932$.

43) Tayarani-Najaran Z, Hajian Z, Mojarrab M, Emami SA. Cytotoxic and apoptotic effects of extracts of Artemisia ciniformis Krasch. and Popov ex Poljakov on K562 and HL-60 cell lines. Asian Pac J Cancer Prev. 2014; 15(17): 7055-9. doi: 10.7314/APJCP.2014.15.17.7055. PMID: 25227790.

44) Miller LH, Su X. Artemisinin: discovery from the Chinese herbal garden. Cell. 2011; 146(6): 855-8. doi: 10.1016/j.cell.2011.08.024. PMID: 21907397, PMCID: PMC3414217.

45) Mueller MS, Runyambo N, Wagner I, Borrmann S, Dietz K, Heide L. Randomized controlled trial of a traditional preparation of Artemisia annua L. (Annual Wormwood) in the treatment of malaria. Trans R Soc Trop Med Hyg. 2004; 98(5): 318-21. doi: 10.1016/j.cell.2011.08.024. PMID: 15109558.

46) Fairhurst RM, Nayyar GM, Breman JG, Hallett R, Vennerstrom JL, Duong S, et al. Artemisinin-resistant malaria: research challenges, opportunities, and public health implications. Am J Trop Med Hyg. 2012; 87(2): 231-41. doi: 10.4269/ajtmh.2012.12-0025. PMID: 22855752, PMCID: PMC3414557.

47) Chrubasik C, Jacobson RL. The development of artemisinin resistance in malaria: reasons and solutions. Phytother Res. 2010; 24(7): 1104-6. doi: 10.1002/ptr.3133. PMID: 20578122.

48) Miraj S, Kiani S. Astragalus membranaceus: A review study of its anti-carcinoma activities. Der Pharmacia Lettre. 2016, 8(6): 59-65. 